\title{
Influences of metal magnetic state and MIS structure composition on magnetoimpedance effect caused by interface states
}

\author{
D.A. Smolyakov ${ }^{*}, 1$, A.S. Tarasov ${ }^{1}$, I.A.Yakovlev ${ }^{1,2}$, A.N. Masyugin ${ }^{2}$, M.N. Volochaev ${ }^{1,2}$, \\ N.N. Kosyrev ${ }^{1}$ and N.V.Volkov ${ }^{1}$. \\ ${ }^{1}$ Kirensky Institute of Physics, Krasnoyarsk, 660036, Russia \\ ${ }^{2}$ Reshetnev Siberian State University of Science and Technology, Krasnoyarsk, 662850
}

*e-mail: sda88@iph.krasn.ru

\begin{abstract}
This article presents the results of the study of metal/insulator/semiconductor (MIS) hybrid structures transport properties in alternating current (ac) mode. We prepared a series of samples with different layers of metal, insulator and semiconductor. Ferromagnetic $\mathrm{Fe}$ and non-magnetic $\mathrm{Cu}$ and $\mathrm{Mn}$ were chosen as metals, the insulators were $\mathrm{SiO}_{2}$ and $\mathrm{Al}_{2} \mathrm{O}_{3}, \mathrm{Si}$ substrates of $\mathrm{n}$ - and $\mathrm{p}$-type were used as semiconductors. Temperature dependence of real part of the impedance showed peculiar peaks below $40 \mathrm{~K}$ for different combinations of metals, insulators and semiconductors. For all samples the effect of magnetic field on the transport properties was studied. At low temperatures, the magnetic field shifts peaks toward higher temperatures. Metal magnetic state doesn't significantly affect this phenomenon. Changing the type of the insulator and its thickness also didn't cause any significant effect. The effect was observed for samples with different composition. However, the type of conductivity of the substrate, as well as the type of-metal, determines the value of magnetoimpedance (MI). The main role in the magnetoimpedance effect is played by recharging of energy states localized at insulator/semiconductor interface. This mechanism allows obtaining MI effect even in "nonmagnetic" MIS structures; MI can be either positive or negative, depending on temperature and frequency. We suggest that the observed ac magnetotransport phenomena could be used for creating magnetic field sensors, working on new principles.
\end{abstract}

Magnetoresistance (MR) and magnetoimpedance (MI) in different materials and structures attract scientific attention from both the point of view of fundamental research and from the point of view of application in manufacturing new electronic devices. Although the effect was found more than half a century ago [1], it attracts strong interest even in these days. Study of structures with the high sensitivity of impedance to an external field is one of the constantly developing areas [2, 3], magnetoresistance are used in magnetic memory slots, different sensors and magnetic antennas [4].

Magnetoimpedance effect is classical explained on the basis of the skin effect - the dependence of the skin layer thickness on the effective magnetic permeability in soft magnetic material [5]. As we reported before [6] MI effect may appear in MIS hybrid structures, due to the presence of interface states at the insulator/semiconductor interface, which participate in the recharging processes when ac voltage is applied to a structure. Mainly the effect of the magnetic field is connecting with a shift of the levels of

a)

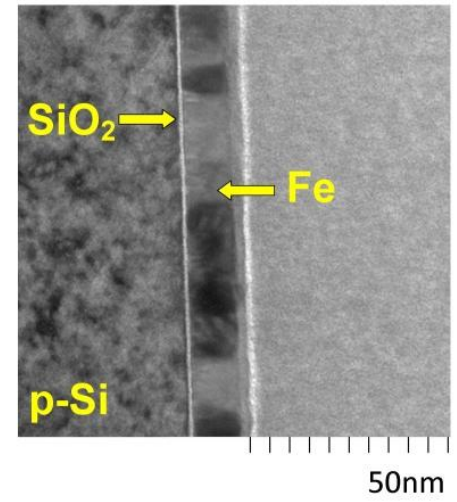

b)

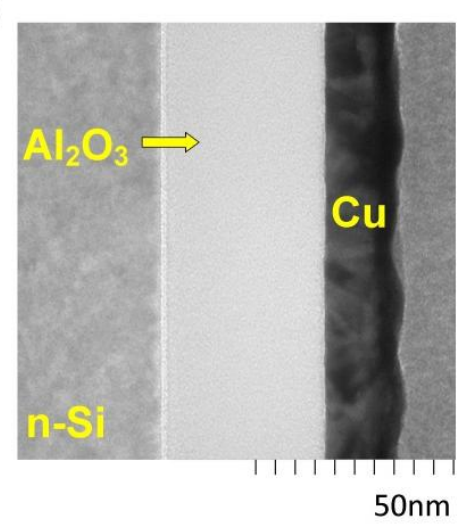

surface states at the insulator/semiconductor interface. Thus, the magneto-impedance effect arises on completely new principles.

In the modern world, silicon are the main component of most semiconductor devices, circuits and microarrays [7]. Thus, the use of silicon-based structures is promising in terms of the effective introduction of new devices into modern electronics [8].

These reasons prompted us to study transport properties of silicon-based hybrid structures under various external influences.

Earlier we have already investigated the MIS structures and this work is a logical extension. We detected the effect of a magnetic field on the structure properties and the possibility of controlling the magnetoresistance value via the bias voltage [6]. Moreover, we found the value of the magnetoresistance reached $10^{8} \%$ [9]. We made assumptions that the main role in these effects is played by surface states at the insulator/semiconductor interface.

Fig. 1. Cross-sectional TEM image of the structure interfaces: a) $\mathrm{Fe} / \mathrm{SiO}_{2} / \mathrm{p}$-Si, b) $\mathrm{Cu} / \mathrm{Al}_{2} \mathrm{O}_{3} / n$-Si; c) Field dependences of MOKE signal for MIS samples.

c)

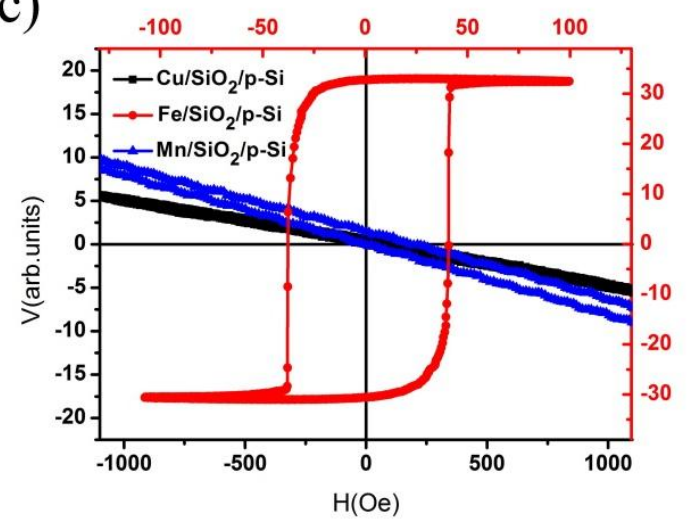




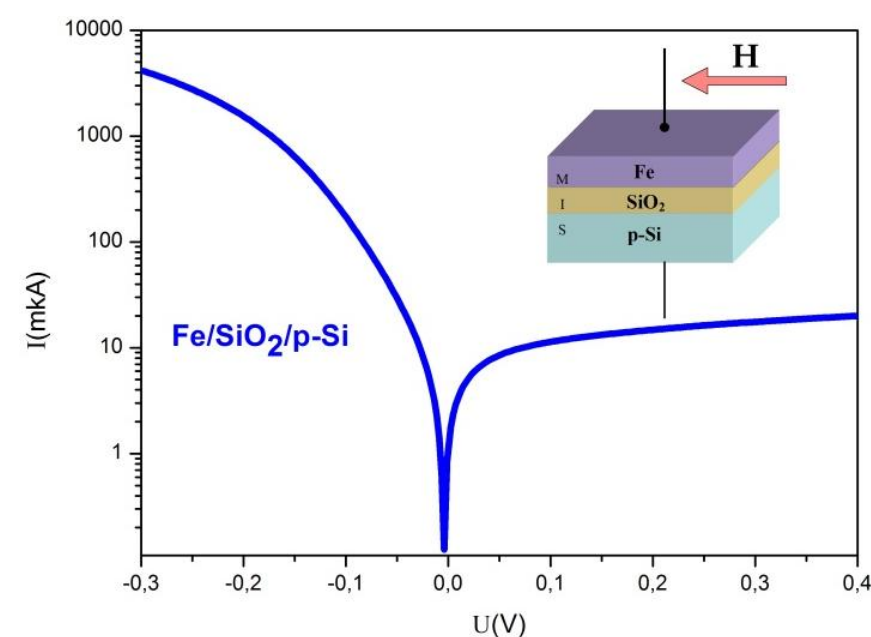

Fig. 2. a) I-V characteristic of $\mathrm{Fe} / \mathrm{SiO}_{2} / \mathrm{p}$-Si MIS structure.

To verify this and exclude the influence of the metal magnetic state, it was decided to make a series of samples with different metal layers.

The structures are MIS diodes with the Schottky barrier. We prepared a series of samples with different layers of metal, insulator and semiconductors. We have chosen ferromagnetic $\mathrm{Fe}$ and nonmagnetic $\mathrm{Mn}$ and $\mathrm{Cu}$ as metals. This was done to determine the contribution of the magnetic state of metal to the magnetoimpedance effect. In addition $\mathrm{SiO}_{2}$ and $\mathrm{Al}_{2} \mathrm{O}_{3}$ were chosen as insulators. This was necessary for determining the role of insulator in the magnetoimpedance properties of the structure. Samples were prepared on a single-crystal $\mathrm{n}$-Si (phosphorus-doped) and $\mathrm{p}$ Si (boron-doped) substrates. The substrate's surfaces were pre-cleaned by the Shiraki method (by chemical etching and long-time annealing at temperatures of $400-650^{\circ} \mathrm{C}$.) [10].

$\mathrm{SiO}_{2}$ layers with a thickness of 1-2 nm were formed on the substrate's surfaces by a chemical method (the substrates were exposed in the aqueous solution of $\mathrm{H}_{2} \mathrm{O}_{2}$ and $\mathrm{NH}_{4} \mathrm{OH}$ in the ratio 1:1:1 for $30 \mathrm{~min}$ at $60^{\circ} \mathrm{C}$ ). $\mathrm{Al}_{2} \mathrm{O}_{3}$ layers were formed by atomic layer deposition (ALD) using a trimethylaluminum precursor (TMA) deionized water and nitrogen carrier gas of 99,9999 purity with growth temperature of $250^{\circ} \mathrm{C}$ on PICOSUN R-200 facility. From 5 to 10 ALD cycles were made in order to obtain the thickness of $50-100 \mathrm{~nm}(9,45 \mathrm{~nm}$ per cycle). Metal films with thicknesses of 10-15 nm were deposited by thermal evaporation under ultrahigh vacuum conditions with sputtering rate of $0.25 \mathrm{~nm} / \mathrm{min}$. Base pressure in "Angara" chamber [11] was $6.5 \times 10^{-8}$ Torr. All the fabricated structures were characterized by cross-sectional transmission electron microscopy (TEM). Magnetic properties were checked by magneto-optical Kerr effect (MOKE) with the help of NanoMOKE 2 setup.

Figure 1 presents cross-sectional TEM image of $\mathrm{Fe} / \mathrm{SiO}_{2} / \mathrm{p}-\mathrm{Si}$ (a) and $\mathrm{Cu} / \mathrm{Al}_{2} \mathrm{O}_{3} / \mathrm{n}-\mathrm{Si}$ (b) structure interfaces. One can see that the structure layers are fairly smooth, with well-defined borders without interdiffusion. Also metal films are polycrystalline with grain size about $10-15 \mathrm{~nm}$. Crosssectional TEM images were taken for the entire series of samples and the results were similar to those shown in the figure. Field dependences of MOKE signal are shown at Figure 1c, from where one can conclude that $\mathrm{Fe}$ is ferromagnetic, $\mathrm{Mn}$ and $\mathrm{Cu}$ are nonmagnetic.
The impedance measuerments were performed by twoprobe method. Ohmic contacts were formed on the metal film using silver epoxy and at the bottom of the Si substrate by indium alloying. The contact pads area was $1 \mathrm{~mm}^{2}$. I-V characteristics of prepared MIS structure (Fig. 2) shows rectifying behavior that indicate forming of space chargeregion in silicon and consequently Schottky barrier at the interface. The impedance measurements were conducted in temperature range of $4.2-350 \mathrm{~K}$ with application of external magnetic field $H$ using Agilent E4980A LCR-meter, helium cryostat and electromagnet [12]. The ac current frequency lied in the range from $20 \mathrm{~Hz}$ to $2 \mathrm{MHz}$; the applied magnetic field values were up to $8 \mathrm{kOe}$. The device is schematically illustrated in inset of Fig. 2.

We firstly measured temperature dependences of the real and imaginary parts of the impedance. Below $40 \mathrm{~K}$, an intensive peak on $R(T)$ curve was found for all samples regardless of the layers composition. Obtained data can be interpreted in terms of admittance spectroscopy measurements [13]. By this technique measured the thermal emission rate at a point of the space-charge region located very close to the crossing point of the Fermi level with the energy level of the surface centers, localized at insulator/semiconductor interface.

a)

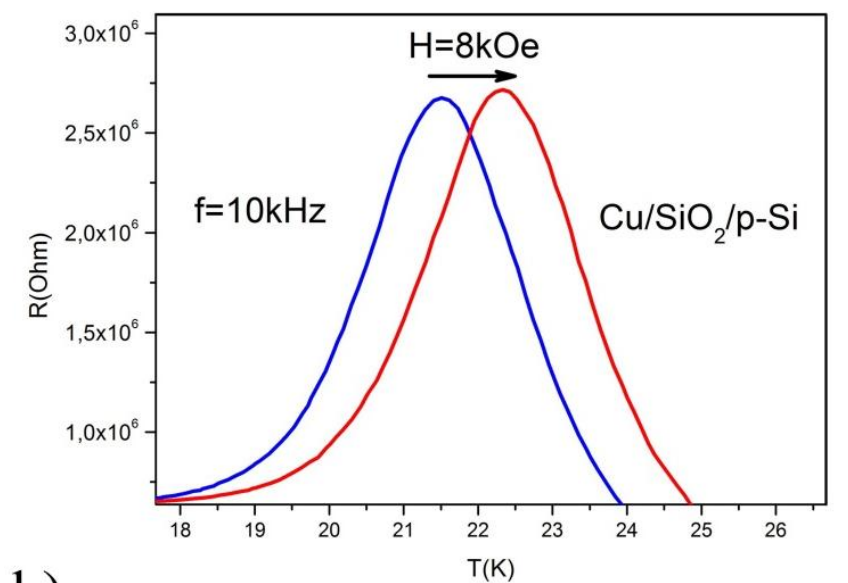

b)

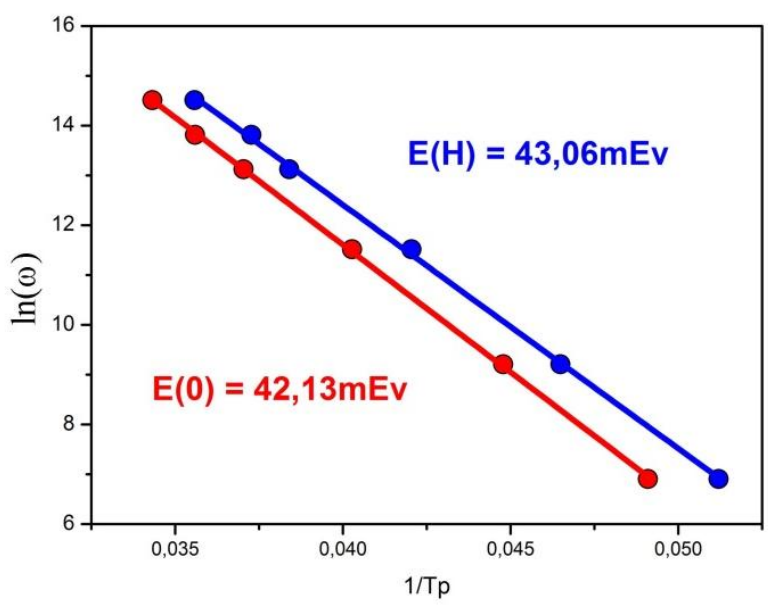

Fig. 3. a) Temperature dependence of the real part of the impedance at $\mathrm{H}=0$ and $\mathrm{H}=8 \mathrm{kOe}$ for $a \mathrm{Cu} / \mathrm{SiO} / \mathrm{p}-\mathrm{Si}, \mathrm{b}) \ln (\omega)$ vs reciprocal temperature peak for determining energy levels, of the interface states for a $\mathrm{Cu} / \mathrm{SiO}_{2} / p$-Si sample. 
In case of MIS structure, process of recharge of localized interface states should be considered as a sequence of capture-emission processes of majority carriers from/to the allowed silicon band and from/to the metal electrode. $R(T)$ peaks appear due to delays of recharging processes.

Since the main purpose was to investigate influence of the magnetic field on transport properties of structures with different composition, we applied an external magnetic field to the samples. Figure $3 \mathrm{a}$ demonstrates temperature dependence of real part of the impedance $R(T)$. Pronounced peaks shifts towards higher temperatures when magnetic field was applied. Shifting of $R(T)$ curve features is apparently related to magnetic field effect on the electronic structure of interface states. We suggest that magnetic field causes the shifting of energy levels of the interface states toward higher energies (toward the center of band gap of the semiconductor).

In addition, magnetic field shifts the peak, regardless of which metal was used during fabrication of the samples. The peak shifts by a value from $0.5 \mathrm{~K}$ to $2 \mathrm{~K}$ for all samples. This fact allows us to say that this effect does not depend on whether the metal is ferromagnetic or not. In our case, for ferromagnetic $\mathrm{Fe}$, and for nonmagnetic $\mathrm{Cu}$ and $\mathrm{Mn}$, effect was approximately the same.

Table I. Parameters of Samples

\begin{tabular}{c|c|c|c} 
Sample & $\begin{array}{c}\text { Energy } \\
(\mathrm{H}=0), \mathrm{mEv}\end{array}$ & $\begin{array}{c}\text { Energy } \\
(\mathrm{H}=8 \mathrm{kOe}), \\
\mathrm{mEv}\end{array}$ & $\mathrm{dE}, \mathrm{mEv}$ \\
\hline \hline $\mathrm{Cu} / \mathrm{SiO}_{2} / \mathrm{p}-\mathrm{Si}$ & 42,13 & 44,06 & 1,93 \\
$\mathrm{Fe} / \mathrm{SiO}_{2} / \mathrm{p}-\mathrm{Si}$ & 43,18 & 43,58 & 0,40 \\
$\mathrm{Mn} / \mathrm{SiO}_{2} / \mathrm{p}-\mathrm{Si}$ & 43,33 & 43,76 & 0,43 \\
\hline $\mathrm{Cu} / \mathrm{SiO}_{2} / \mathrm{n}-\mathrm{Si}$ & 43,11 & 43,29 & 0,18 \\
$\mathrm{Fe} / \mathrm{SiO}_{2} / \mathrm{n}-\mathrm{Si}$ & 42,51 & 42,52 & 0,01 \\
$\mathrm{Mn} / \mathrm{SiO}_{2} / \mathrm{n}-\mathrm{Si}$ & 41,92 & 42,08 & 0,16 \\
\hline
\end{tabular}

For all the samples we calculated the energy of the interface states in zero and non-zero magnetic field using relation $\ln (\omega)=\ln \left(1 / \tau_{0}\right)-E_{s} /\left(k_{B} T_{p}\right)$ [14]. $T_{p}$ is a peak position on $R(T)$ curve at fixed $\omega$. By making a linear fit of the experimental $\ln (\omega)$ vs $1 / T_{p}$ dependence, from the slope of the fitting line, we estimated the energy of the interface states. Such fitting is presented in Fig. $3 b$ for $\mathrm{Cu} / \mathrm{SiO}_{2} / \mathrm{p}-\mathrm{Si}$ at $\mathrm{H}=8 \mathrm{kOe}$ and $\mathrm{H}=0$. Energy values for different samples can be seen in Table 1 First of all, calculated values of energy states without magnetic field are very close to ionization energies for doping impurities. For phosphorus and boron the energy is $46 \mathrm{meV}$ and $44 \mathrm{meV}$, respectively [15]. It is logical to assume that interface states levels in our structures are formed by phosphorus and by boron for $\mathrm{n}$ - and p-type substrates.

Moreover, as can be seen from the Table 1, a change in the energy of the surface states in magnetic field is observed for all samples. From that we conclude the shift of a feature on $R(T)$ in magnetic field does not related to ferromagnetic state of a metal. Changes of energy for structures with different metal layers has the same order of magnitude. Even for nonmagnetic $\mathrm{Cu}$ the effect is noticeably higher than for structure with ferromagnetic Fe layer.

\section{a)}

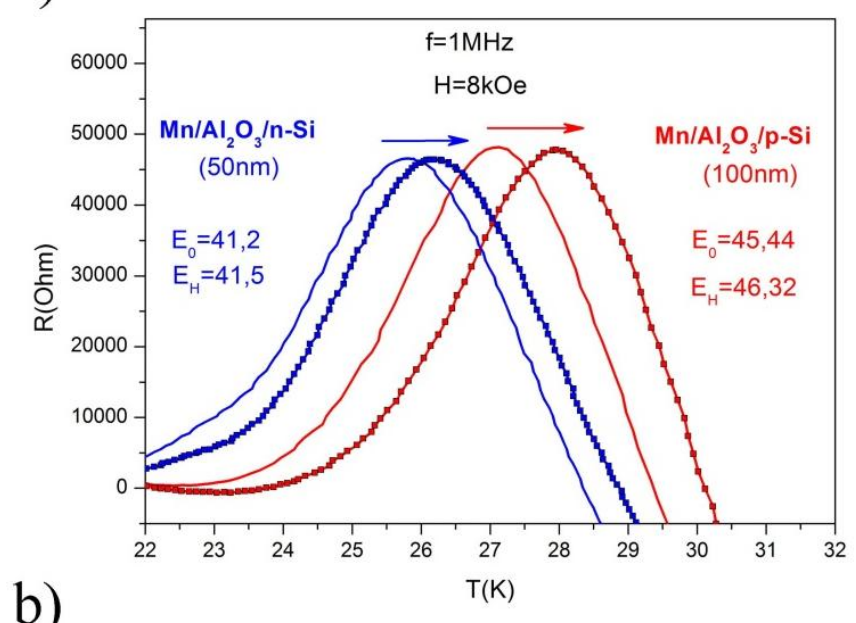

b)

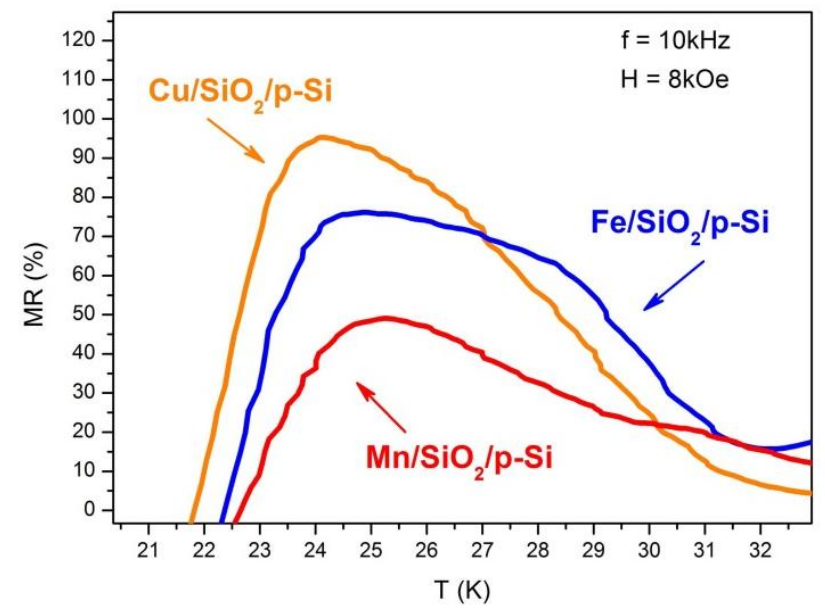

Fig. 4. a) Temperature dependence of the real part of impedance at $\mathrm{H}=0$ (line) and $\mathrm{H}=8 \mathrm{kOe}$ (line with solid squares) for $\mathrm{Mn} / \mathrm{Al}_{2} \mathrm{O}_{3} / \mathrm{p}$-Si and $\mathrm{Mn} / \mathrm{Al}_{2} \mathrm{O}_{3} / \mathrm{n}$-Si, b) magnetoresistance of MIS-structures with p-type substrates.

However, it can be seen that for samples with n-type substrates, the energy change is much less than for p-type. This is probably related to the properties of surface states, formed by donors or acceptors, phosphorus or boron, respectively. Also, anomalous values of the energy change were observed for $\mathrm{Cu} / \mathrm{SiO}_{2} / \mathrm{p}-\mathrm{Si}$ and $\mathrm{Fe} / \mathrm{SiO}_{2} / \mathrm{n}-\mathrm{Si}$. We connect this with the quality of the insulator/semiconductor interface. Additional studies related to the structural and chemical quality of the border are needed.

In addition to the above, the peak shift under the action of the magnetic field did not depend on the type of the insulator. Figure $4 \mathrm{a}$ shows $R(T)$ curves for $\mathrm{Mn} / \mathrm{Al}_{2} \mathrm{O}_{3} / \mathrm{n}-\mathrm{Si}$ and $\mathrm{Mn} / \mathrm{Al}_{2} \mathrm{O}_{3} / \mathrm{p}-\mathrm{Si}$. When $\mathrm{Al}_{2} \mathrm{O}_{3}$ is used, the $\mathrm{R}(\mathrm{T})$ peak shifting is observed as for $\mathrm{SiO}_{2}$. Like for the previous samples, the energy of the interface states was also calculated for these structures. Moreover, for these structures, we used $\mathrm{Al}_{2} \mathrm{O}_{3}$ with thicknesses of 50 and $100 \mathrm{~nm}$, and the change of thickness didn't significantly affect $R(T)$ features changing in magnetic field. Our ongoing studies of the epitaxial $\mathrm{Fe}_{3} \mathrm{Si} / \mathrm{p}$-Si structure showed that such a change in the impedance upon exposure to a magnetic field is observed even without a dielectric layer. All of the above allows us to trace the tendency. The thickness and material of the dielectric layer does not exert a determining influence on the energy levels of interface states shifting and the 
magnetoimpedance correspondingly. While the type of conductivity of the substrate, as well as the type of metal, determines the magnitude of the MI effect.

If we consider the effect from the point of view of device manufacturing, these structures show enough of magnetic field sensitivity. Magnetoresistance values obtained for the samples varied from 50 to $100 \%$. Magnetoresistance is defined as $M R=100 \% \times((R(H)-R(0)) / R(0))$.

Figure $4 \mathrm{~b}$ demonstrates $\mathrm{MR}$ for different samples at $\mathrm{H}=8 \mathrm{kOe}$. The maximum MR value of $100 \%$ is obtained for $\mathrm{Cu} / \mathrm{SiO}_{2} / \mathrm{p}-\mathrm{Si}$, minimum value of $50 \%$ for $\mathrm{Mn} / \mathrm{SiO}_{2} / \mathrm{p}-\mathrm{Si}$ and value of $80 \%$ for $\mathrm{Fe} / \mathrm{SiO}_{2} / \mathrm{p}$-Si. Samples with p-type substrates were chosen because the change of the interface states energy is higher for $p$-type substrates than for n-type substrates. Thus, the use of such structures is quite promising for manufacturing, for example, magnetic sensors which prototypes already exist.

In this paper we presented the results of measurements of the ac transport properties of silicon-based hybrid structures with different composition under the action of a magnetic field. It was found that magnetic field affects the surface states located at the insulator/semiconductor interface. Peak shifting in the temperature dependence of the real part of the impedance is observed. The magnetic state of metals did not affect this effect it was observed for all samples. Also, the type of insulator and its thickness did not make significant changes. Nevertheless, the type of conductivity of the substrate, as well as the type of metal, determines the magnitude of the MI effect. This is probably related to the properties of the surface states formed by donors or acceptors. It can be assumed that using the technology of ion implantation, it is possible to synthesize nonmagnetic structures with various dopants, in which the effect of MI will be observed at different temperatures.

\section{Acknowledgements}

The reported study was funded by RFBR according to the research project № 18-32-00035. The work was partially supported by Russian Foundation for Basic Research, Government of Krasnoyarsk Territory, Krasnoyarsk Region Science and Technology Support Fund by project № 18-42243022.

\section{References}

1. Harrison E.P., Turney G.L., Rowe H. An impedance magnetometer // Nature. - 1935. - № 135. - C. 961

2. Morikawa T., Nishibe Y., Yamadera H., Nanomura Y., Takeuchi M., Taga Y. Giant Magneto-Impedance in Layered thin films // IEEE Transactions on Magnetics. - 1997. - T 33. - № 5

3. Chiriac H., Herea D.D., Corodeanu S. Microwire array for giant magneto-impedance detection of magnetic particles for biosensor prototype // J. Magn. Magn. Mater. . - 2007. - № 311. - C. $425-428$

4. A. Fert. Phys. Usp. 51(2008)

5. Knobel M., Vázquez M., Kraus L. Giant magnetoimpedance // Handbook of Magnetic Materials. 2003. - № 15. - C. 497 - 563

6. N.V. Volkov, A.S. Tarasov, D. A. Smolyakov, A. O. Gustaitsev, V. V.Balashev, and V. V. Korobtsov. The biascontrolled giant magnetoimpedance effect caused by the interface states in a metal-insulator-semiconductor structure with the Schottky barrier // Applied Physics Letters. - 2014. - № 104. - C. 222406 (1-5)

7. Ö. Demircioglu, Ş. Karataş, N. Yıldırım, Ö.F. Bakkaloglu, A. Türüt, Temperature dependent current-voltage and capacitance-voltage characteristics of chromium Schottky contacts formed by electrodeposition technique on n-type $\mathrm{Si}$, J. Alloy. Compd. 509 (2011) 6433-6439

8. T. Wang, et al. AIP Advances 7(2017)

9. N. V. Volkov, A. S. Tarasov, D. A. Smolyakov, A. O. Gustaitsev, M. V. Rautskii, A. V. Lukyanenko, M. N. Volochaev, S. N. Varnakov, I. A. Yakovlev and S. G. Ovchinnikov. Extremely high magnetic-field sensitivity of charge transport in the $\mathrm{Mn} / \mathrm{SiO} 2 / \mathrm{p}-\mathrm{Si}$ hybrid structure // AIP Advances. - 2017. - № 7. - C. 015206 (1-13)

10. A. Ishizaka and Y. Shiraki,J. Electrochem. Soc.133, 666 (1986).

11. S.N. Varnakov, A.A. Lepeshev, S.G. Ovchinnikov, A.S. Parshin, M.M. Korshunov, P. Nevoral, Automation of technological equipment for obtaining multilayer structures in an ultrahigh vacuum, Instrum. Exp. Tech. 47 (2004) 839843.

12. Volkov N.V., Tarasov A.S., Eremin E.V., Eremin A.V., Varnakov S.N., Ovchinnikov S.G. Frequency-dependent magnetotransport phenomena in a hybrid $\mathrm{Fe} / \mathrm{SiO} 2 / \mathrm{p}-\mathrm{Si}$ structure, Journal of Applied Physics 112 (12) (2012) 123906

13. Characterization of the DX centers in AIGaAsSi by admittance spectroscopy J. Appt. Phys. 69 (8), 15 April 1991 14. D. L. Losee,J. Appl. Phys.46, 2204 (1975).

15. S. M. Sze, Kwok K. Ng., Physics of Semiconductor Devices (Wiley-Interscience, New Jersey, 2007). p. 23 